ing the theorem, but Kummer's paper of 1857 is not even listed, although some of the results are mentioned in connection with Noguès' report on the contents of Mordell's pamphlet of 1921, (French translation, 1929).

Chapter XI was designed to cover the period from 1910-1931, but among the nine reports on articles given therein all but two of the authors are French! No mention is made of Frobenius' article of 1914, (except in the report on Mordell), or of the interesting results obtained by Furtwängler, Pollaczek, Beeger, or Morishima, during the period treated.

It appears that little if any use was made by Noguès in the preparation of his book of the Report on Algebranc Numbers, II, Bulletin of the National Research Council, 1928, by G. E. Wahlin and the present writer, which treats the class number of an algebraic field and Fermat's Last Theorem.

I have already referred to the fact that incorrect proofs of the Last Theorem have been reproduced by our author in some cases without comment by him. With one exception, this should cause little difficulty to the reader, as the errors may be readily detected. In the case of the report on Démonstration $d u$ Théorème de Fermat, by E. Fabry, (1913), however, the error in his supposed proof that the Fermat relation is impossible in Case I, lies considerably below the surface. It was pointed out by Mirimanoff in the Comptes Rendus, vol. 157 (1913), pp. 491-492.

The principal value of the book, in my opinion, lies in the fact that the author has given extensive accounts of the work of Legendre, Dirichlet, Lamé, V. Lebesgue, and Mirimanoff.

\title{
H. S. VANDIVER
}

Les Principes de la Mécanique Quantique. By P. A. M. Dirac. Traduit par Al. Proca et J. Ullmo. Paris, Les Presses Universitaires de France, 1931. viii +314 pp.

This translation of Dirac's Principles of Quantum Mechanics appears as Vol. 21 of the series Conférences-Rapports de Documentation sur la Physique. The English edition was amply reviewed in this Bulletin, vol. 37 (1931), pp. 495-496, and it is only necessary to say here that the French edition contains a useful appendix of nine pages by one of the translators (Al. Proca) giving a summary account of the most important properties of the Poisson and Lagrange brackets of classical mechanics. The translation has been carefully done, and the printing and general appearance of the book are admirable.

F. D. Murnaghan 